\title{
A Method to Enhance the Data Transfer Rate of Phase Change Recording Media
}

\author{
Tung-Ti Yeh*, T.E. Hsieh*, and Han-Ping D. Shieh\# \\ *Department of Materials Science and Engineering, National Chiao-Tung University, \\ 1001, Ta-Hsueh Rd., Hsinchu 30050, Taiwan; ROC. \\ \#Institute of Electro-Optical Engineering, National Chiao-Tung University, \\ 1001, Ta-Hsueh Rd., Hsinchu 30010, Taiwan, ROC.
}

\begin{abstract}
Nitrogen doping was applied to enhance the velocity of amorphous-crystalline phase transformation. The nitrogen doping was able to produce tiny nitride precipitates uniformly distributed in the recording layer and provided numerous preferential sites for amorphous-crystalline transition. At the doping condition of $\mathrm{N}_{2} / \mathrm{Ar}$ ratio $=3 \%$, the recrystallization velocity of GeInSbTe phase change recording media was increased up to 1.6 times without severely damaging the signal jitter values.
\end{abstract}

Keywords: Nitrogen doping, phase transformation, recrystallization velocity, GeInSbTe.

\section{INTRODUCTION}

The development of optical recording media using phase change materials always pursues large storage capacity and high data transfer rate. The phase change recording materials with higher amorphous-crystalline transition speed are hence required. Recently the eutectic Sb-Te system, or called the fast-growth phase change alloy, has drawn a lot of attentions since it possesses good signal properties and high recrystallization speed when employing short wavelength laser and pickup head with high numerical aperture (NA) lens. The eutectic Sb-Te system is termed as the fast-growth material since its recrystallization is initiated from the crystalline-amorphous interface and the amorphous mark shrinks as the grain growth propagates toward the center of the mark ${ }^{(1-4)}$, as illustrated in Fig. 1(a). In this work, we doped nitrogen in the eutectic GeInSbTe recording layer of the optical disks and investigated their signal properties and data transfer rate by using dynamic test. Transmission electron microscopy (TEM) was utilized to examine the microstructure change induced by nitrogen doping and its effects on the data transfer rate and/or recrystallization speed were discussed. 


\section{THE MODEL OF RECRYSTALLIZATION PROCESS}

Figure 1(b) illustrates the model applied to enhance the velocity of phase transformation. Nitrogen doping generates numerous nanometer-scale precipitates uniformly distributed in the recording layer. They provide the preferential sites for amorphous-crystalline transition so that the edge of marks is not the only site to initiate the recrystallization of recording media. The tiny precipitates not only induce the heterogeneous nucleation, they also shorten the distance of grain growth required to complete the transition. Hence the velocity of amorphous-crystalline transition is increased.

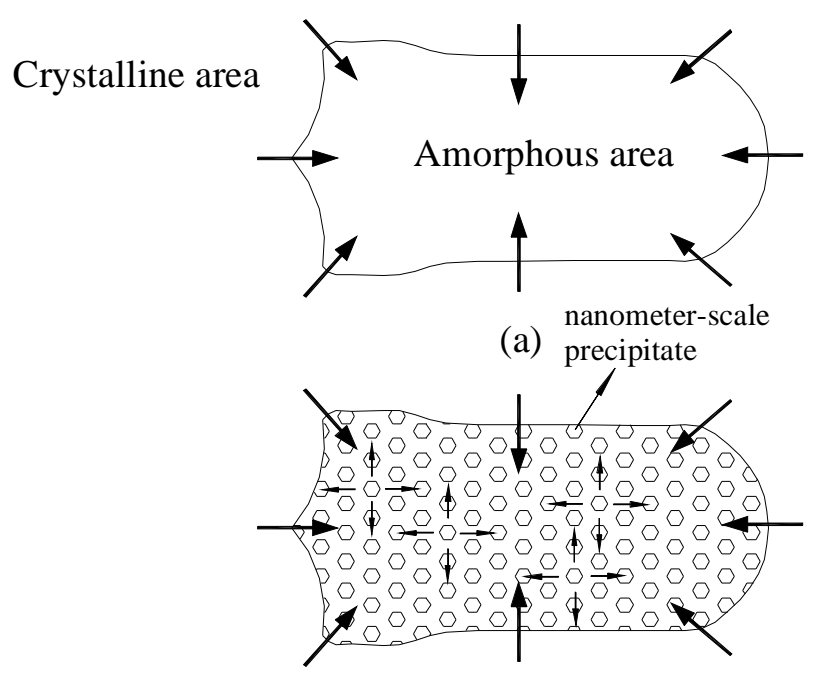

(b)

Figure 1. Schematic illustration of the recrystallization process. (a) In doping-free recording media, amorphous-crystalline transition occurs at the edge of mark; (b) In nitrogen-doped media, the phase transition simultaneously initiates from the edge of mark and precipitate interfaces so as to promote the recrystallization speed.

\section{EXPERIMENTAL}

Figure 2 depicts the multilayer structure of the optical disk prepared in this work for dynamic test. The disk samples were prepared using a SFI sputtering system with background pressure better than $1 \times 10^{-6}$ torr. The multilayer structure was deposited on 0.6-mm-thick polycarbonate (PC) substrate in the sequence of $\mathrm{ZnS}^{-\mathrm{SiO}_{2}}$ (55 $\mathrm{nm}) /$ GIST-(N) $)_{x}(16 \mathrm{~nm}) / \mathrm{ZnS}-\mathrm{SiO}_{2}(11 \mathrm{~nm}) / \mathrm{Al}-\mathrm{Cr}(133 \mathrm{~nm})$. During the deposition of recording layer, the $\mathrm{N}_{2} / \mathrm{Ar}$ flow ratio was adjusted at values of $0 \%, 0.5 \%, 1 \%, 3 \%, 5 \%$, and $10 \%$ respectively in order to obtain the doped specimens with various nitrogen contents. The sputtering conditions and designated sample numbers are listed in Table 1 . 


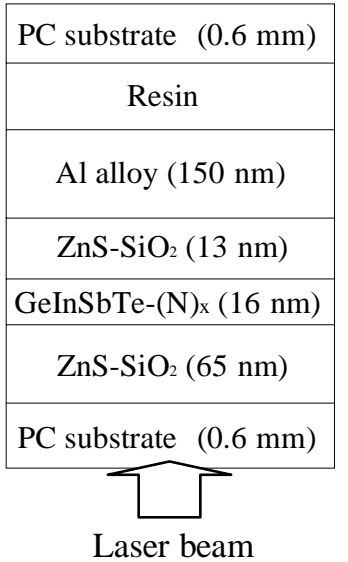

Figure 2. The structure of optical disk sample.

Table 1. Sputtering conditions and designated sample numbers.

\begin{tabular}{|c|c|c|c|c|}
\hline Sample No. & $\mathrm{N}_{2}$ / Ar ratio & Target type & Sputtering pressure (m Torr) & Sputtering power (W) \\
\hline \hline--- & 0 & ZnS-SiO2 & 3 & $250(\mathrm{RF})$ \\
\hline--- & 0 & Al-Cr & 3 & $400(\mathrm{DC})$ \\
\hline N000 & 0 & GeInSbTe & 3 & $50(\mathrm{RF})$ \\
\hline N005 & $0.5 \%$ & GeInSbTe & 3 & $50(\mathrm{RF})$ \\
\hline N010 & $1.0 \%$ & GeInSbTe & 3 & $50(\mathrm{RF})$ \\
\hline N030 & $3.0 \%$ & GeInSbTe & 3 & $50(\mathrm{RF})$ \\
\hline N050 & $5.0 \%$ & GeInSbTe & 3 & $50(\mathrm{RF})$ \\
\hline N100 & $10.0 \%$ & GeInSbTe & 3 & $50(\mathrm{RF})$ \\
\hline
\end{tabular}

Ge:In:Sb:Te = 4.5:4:65.95:25.55 (at weight percent )

After initialized at the condition of $1000 \mathrm{~mW}$ laser power, $50 \mu \mathrm{m}$ per revolution and $2.0 \mathrm{~m} / \mathrm{sec}$ linear velocity, the disk were sent to a dynamic tester (DDU1000, PULSTEC Co.) having a pickup head with 650 nm laser diode and a 0.6-NA objective lens to evaluate their signal properties. At the beginning of dynamic test, the disks were written at various laser power ( 7 to $15 \mathrm{~mW})$ with fixed erasing power $\left(P_{e}=6 \mathrm{~mW}\right)$ and reading power $\left(P_{r}=0.7 \mathrm{~mW}\right)$ under various linear velocities in order to identify the appropriate writing power $\left(P_{w}\right)$. Then the track recorded with $8 \mathrm{~T}$ signals was erased by irradiating a DC laser beam with various laser power ( 3 to $7 \mathrm{~mW}$ ) at a linear velocity same as that for recording. The carrier-to-noise ratio (CNR) was measured before and after the erasing process to determine the attenuation of $8 \mathrm{~T}$ signal carrier and the value of the attenuation of $8 \mathrm{~T}$ signal carrier is defined as DC erasability.

The method report by Chen et al. ${ }^{(5,6)}$ was adopted to prepare the plan-view TEM (PTEM) specimens. After removing the PC substrate, the disk sample was cut into small pieces using a scissor. A $3 \mathrm{M}$ tape was applied on the disk to peel off the Al-Cr reflection layer. After dissolution of the PC substrate by $\mathrm{CH}_{2} \mathrm{Cl}_{2}$ solution, the specimen was 
mounted on the copper mesh and transferred to TEM (Philips Tecnai 20 TEM) for microstructure observation.

\section{RESULTS AND DISCUSSION}

(1) DC erasability: Table 2 shows the DC erasability of $8 \mathrm{~T}$ signals as a function of linear velocity and/or data transfer rate of the optical disks doped at various $\mathrm{N}_{2} / \mathrm{Ar}$ ratios. The outputs of DC laser power were adjusted so that the DC erasability at each linear velocity reached the maximum. As we known, the DC erasability higher than $25 \mathrm{~dB}$ is required for direct overwriting (DOW) of optical disks. From the results of DC erasability test shown in Table 2, the doping-free disk sample just passes the test requirement at the linear velocity below $5.3 \mathrm{~m} / \mathrm{sec}$. However, the nitrogen-doped samples, for instance, the N030 sample successfully passes the DC erasability test requirement at the linear velocity of $8.8 \mathrm{~m} / \mathrm{sec}$. It means that the recrystallization speed of N030 sample was about 1.6 times higher than that of the sample free of nitrogen. Apparently, suitable amount of nitrogen doping benefited the data transfer rate and/or recrystallization speed of Ge-In-Sb-Te phase change optical disks.

Table 2. DC erasability of optical disks at different linear velocity.

\begin{tabular}{|c|c|c|c|c|c|}
\hline Data transfer rate (Mbps) & 11.08 & 16.62 & 22.16 & 27.70 & 33.24 \\
\hline Linear velocity (m/sec) & 3.5 & 5.3 & 7.0 & 8.8 & 10.5 \\
\hline \hline Sample & \multicolumn{5}{|c|}{ DC erasability } \\
\hline \hline N000 & 39.89 & 28.03 & $12.38^{*}$ & --- & --- \\
\hline N005 & 48.59 & 43.86 & 34.36 & $16.58^{*}$ & --- \\
\hline N010 & 37.17 & 48.81 & 42.00 & $17.28^{*}$ & --- \\
\hline N030 & 36.75 & 40.36 & 42.34 & 26.00 & $16.91 *$ \\
\hline N050 & $\times$ & $\times$ & $\times$ & $\times$ & $\times$ \\
\hline N100 & $\times$ & $\times$ & $\times$ & $\times$ & $\times$ \\
\hline
\end{tabular}

(2) Jitter values: Figure 3 shows the jitter values of N000 and N030 disk samples. It was found that the jitter values of both samples are quite similar at the same number of DOW cycles. This implies that a relatively small amount of nitrogen doping would not deteriorate signal properties of disks. 


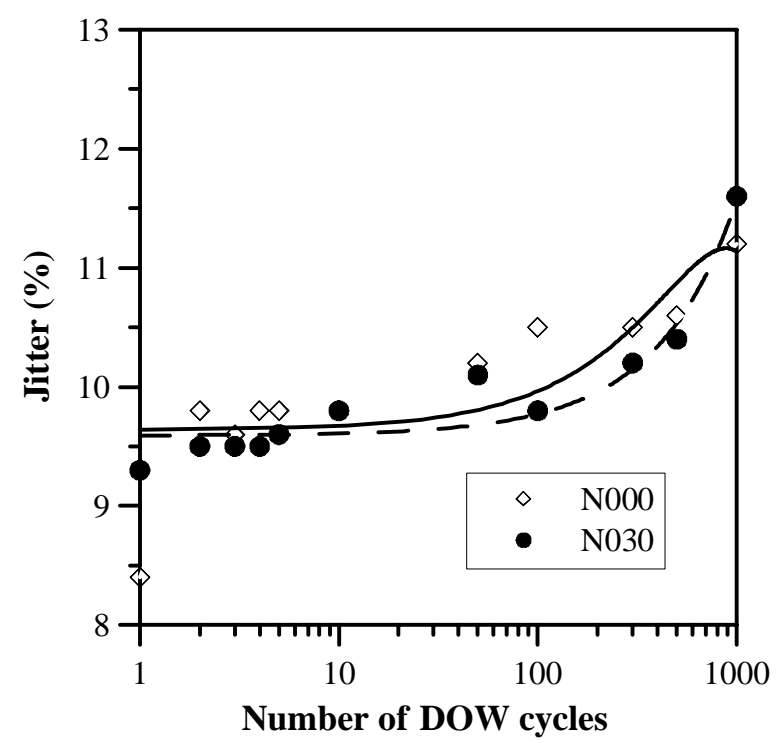

Figure 3. Jitter values versus the number of DOW cycles at the linear velocity of $3.5 \mathrm{~m} / \mathrm{sec}$ for N000 and N030 disk samples.

(3) TEM observation: The micrographs of 8T signal in disk samples of N000 and N030 written at the linear velocity of $7 \mathrm{~m} / \mathrm{sec}$ and $10.5 \mathrm{~m} / \mathrm{sec}$ are shown in Figs. 4(a) and 4(b). The lamellar-like structure commonly seen in the eutectic recording alloy was observed in both of these samples. In addition to the higher degree of structure irregularity, we observed tiny precipitates uniformly distributed in N030 sample at high magnification. These precipitates with size less than $10 \mathrm{~nm}$ were considered to be the nitride compounds, e.g., Ge-N, Sb-N, Te-N, In-N, etc. and were stable under laser irradiation as shown in Fig. 3. Figures 4(c) and 4(d) show the micrographs of residual marks in N000 and N030 samples separately erased at the linear velocities of $7 \mathrm{~m} / \mathrm{sec}$ and $10.5 \mathrm{~m} / \mathrm{sec}$. As shown in Figs. 4(c) and 4(d), the residue of marks can be easily found in the undoped sample while in the nitrogen-doped specimens the residual marks were hardly observed. The eutectic GIST alloy is termed as the fast-growth material since its recrystallization is initiated from the crystalline-amorphous interface and the amorphous mark shrinks as the grain growth propagates toward the center of the mark, as illustrated in Fig. 1(a). The velocity of grain growth derived from the net jump frequency of atoms across the amorphous-crystallization interface can be expressed as ${ }^{(7)}$

$$
V(T)=V_{0} e^{-\frac{E_{a}}{R \Delta T}}\left(1-e^{-\frac{\Delta g^{a c}}{R T}}\right)
$$

where $V_{o}$ is a pre-exponential factor, $E_{a}$ is the activation energy of amorphous-to-crystalline phase transition, $\Delta g^{a c}$ is the free energy difference between an atom in the amorphous state and in the crystalline state, $R$ is the gas constant and $\Delta T$ is the temperature difference between the interface temperature and the glass transition temperature. From Eq. (1), it is known that low $E_{a}$ value benefited amorphous-to-crystalline phase transition, but it seemed deviation for our experiment since we measured five different crystallization temperatures at different heating rates $(5,10,20,40$ and 80 


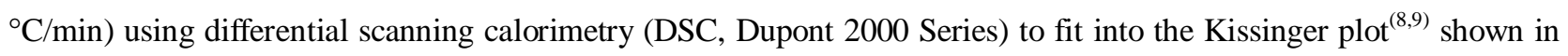
Eq. (2) to calculate the activation energy for crystallization. The Kissinger equation is

$$
\ln \left(\frac{\alpha}{T_{P}^{2}}\right)=-\left(\frac{E_{a}}{R}\right) T_{P}^{-1}+c
$$

where $\alpha$ is a heating rate, $T_{P}$ is the crystallization temperature, $E_{a}$ is the activation energy for phase transformation, $R$ is the Boltzamann constant and $C$ is a constant. A plot of $\ln \left(\alpha / T_{p}{ }^{2}\right)$ versus $1 / T_{p}$ yields a straight line, as shown in Fig. 5 and the activation energy of amorphous-to-crystalline transformation can be calculated. The activation energies of nucleation for N000, N005, N010 and N030 were $2.462 \mathrm{eV}, 2.521 \mathrm{eV}, 2.642 \mathrm{eV}$ and $1.686 \mathrm{eV}$, respectively, as shown in Fig. 6. In according to the recrystallization model shown in Fig. 1(b), nitrogen doping might generate numerous nanometer-scale precipitates uniformly distributed in the GIST recording layer. In addition to the amorphous-crystalline edge of marks, they were also the preferential sites for amorphous-crystalline transition of recording media. The tiny precipitates not only induce the heterogeneous nucleation, they also shorten the distance of grain growth required to complete the transition.

\section{CONCLUSIONS}

We demonstrated that the nitrogen doping is a promising method to enhance the recrystallization speed and/or data transfer rate of GeInSbTe phase change recording media. The nitrogen doping might produce tiny nitride precipitates uniformly distributed in the recording layer and provide numerous preferential sites for amorphous-crystalline transition. This not only promotes the heterogeneous nucleation, but also shortens the distance of grain growth required to complete the transition. Our experiment found that at the doping $\mathrm{N}_{2} / \mathrm{Ar}$ ratio $=3 \%$, the recrystallization velocity of GeInSbTe phase change recording media was increased up to 1.6 times without severely damaging the signal jitter values.

\section{ACKNOWLEDGEMENT}

This work was supported by the Ministry of Education of the Republic of China with the Academic Center of Excellence in "Photonics Science and Technology for Tera Era" under contract No. 89-E-FA06-1-4. 


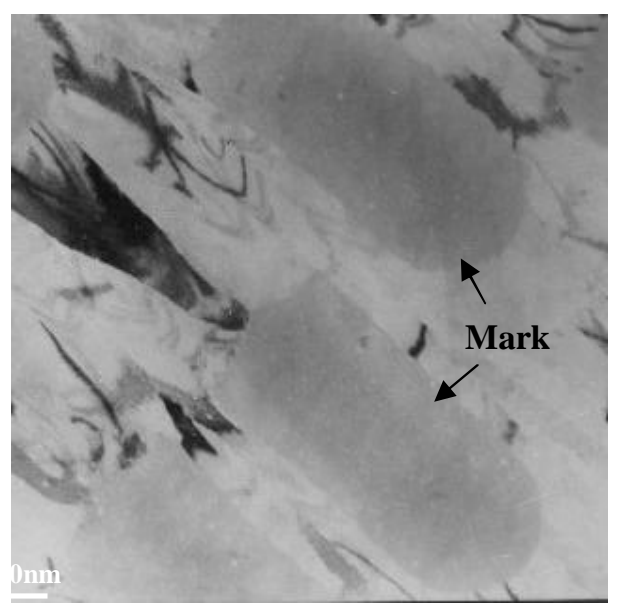

(a)

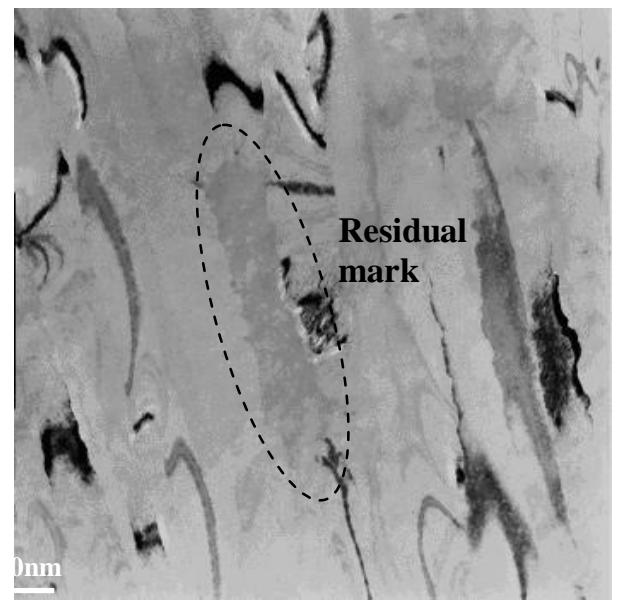

(c)

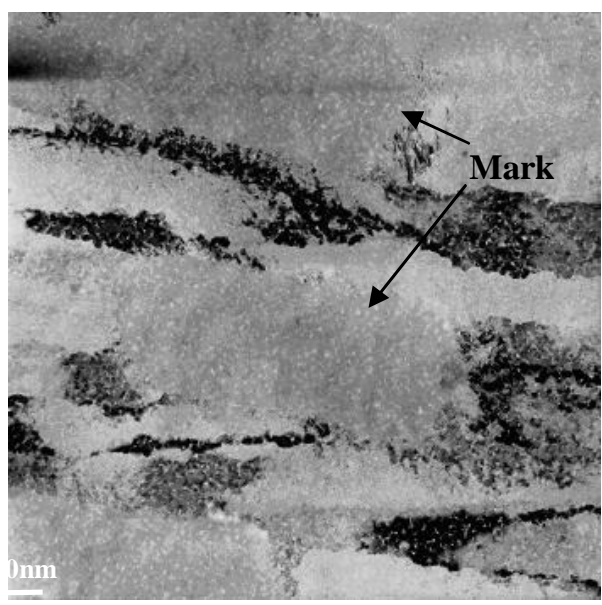

(b)

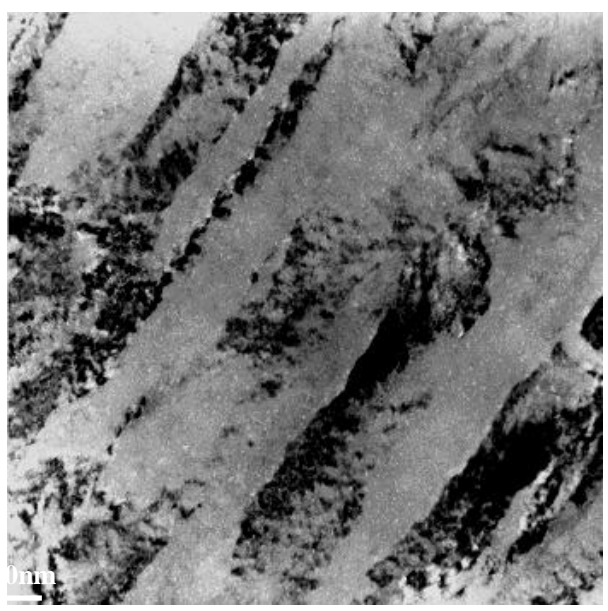

(d)

Figure 4 The micrographs of 8T signal marks of (a) N000 disk sample written at the linear velocity of $7 \mathrm{~m} / \mathrm{sec}$ and (b) N030 disk sample written at the linear velocity of $10.5 \mathrm{~m} / \mathrm{sec}$. The micrographs of residual amorphous marks of (c) N000 disk sample erased at linear velocity of $7 \mathrm{~m} / \mathrm{sec}$ and (d) N030 disk sample erased at linear velocity of $10.5 \mathrm{~m} / \mathrm{sec}$. 


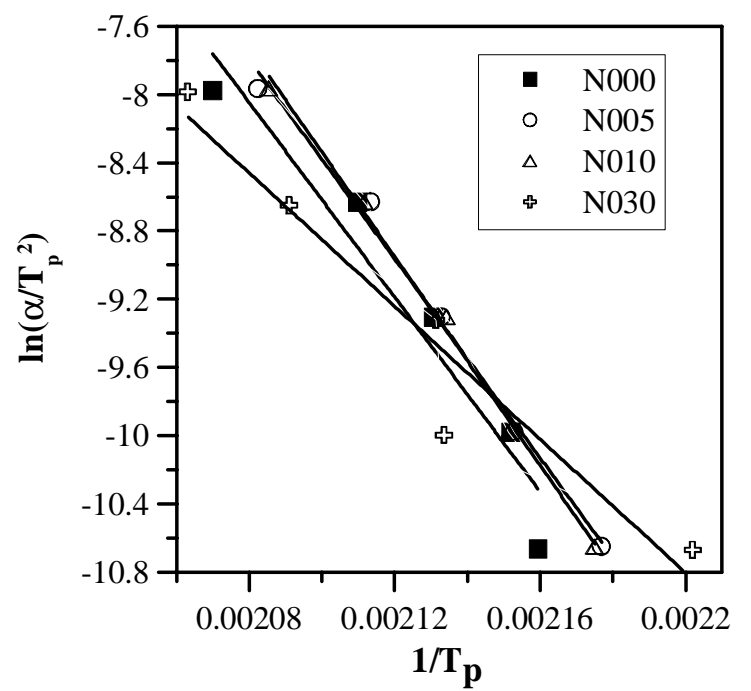

Figure 5. The Kissinger plot of N000, N005, N010, and N030 samples.

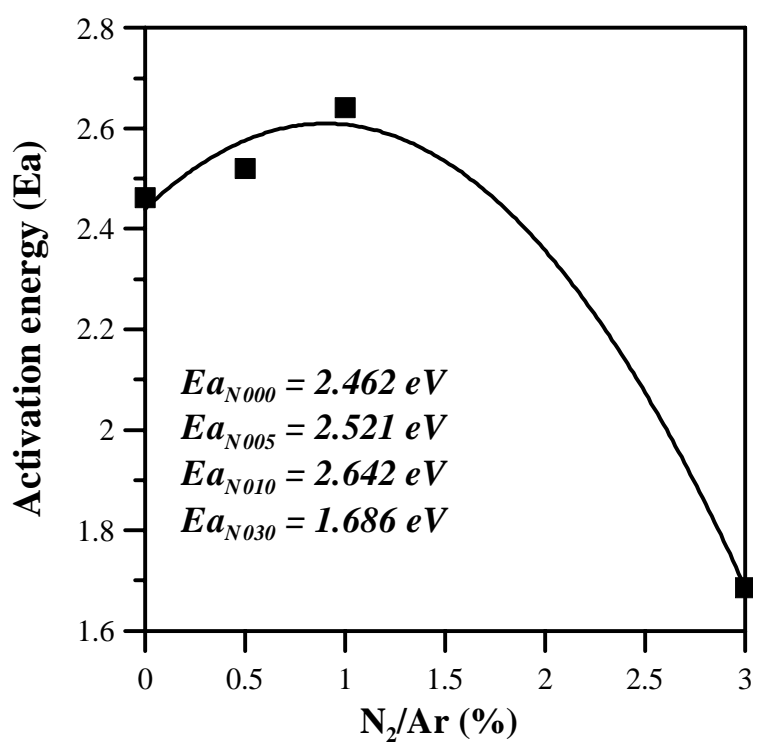

Figure 6. Calculation of activation energy for N000, N005, N010 and N030 samples according to Kissinger plot shown in Fig. 5.

\section{REFERENCES}

1. $\quad$ R. E. Reed-Hill, Physical Metallurgy Principles $2^{\text {nd }}$ ed., Chap. 13.

2. D. A. Porter and K. E. Easterling, Phase Transformations in Metals and Alloys (Chapman \& Hall, New York, 1992) $2^{\text {nd }}$ ed., Chap5.

3. E. R. Meinders, H. J. Borg, M. H. R. Lankhorst, J. Hellmig and A. V. Mijiritskii, J. Appl. Phys., 91 (2002) 9794.

4. L. H. Chou and Y. Y. Chang, Jpn. J. Appl. Phys., 40 (2001) 1272.

5. N. Nobukuni, M. Takashima, T. Ohno and M. Horie, J. Appl. Phys., 78 (1995) 6980. 
6. H. W. Chen, T. E. Hsieh, J. R. Liu and H. P. D. Shieh, Jpn. J. Appl. Phys., 38 (1999) 1691.

7. E. R. Meinders, H. J. Borg, M. H. R. Lankhorst, J. Hellmig and A. V. Mijiritskii, J. Appl. Phys., 91 (2002) 9794.

8. H. Seo, T. H. Jeong, J. W. Park, C. Yeon, S. J. Kim and S. Y. Kim, Jpn. J. Appl. Phys., 39 (2000) 745.

9. H. E. Kissinger, Anal. Chem., 29 (1957) 1702. 